\title{
Leucemia linfocítica crónica
}

\section{Artículo de Revisión}

Arias-Segura Jorge Omar*, Valero-González José Mauricio**

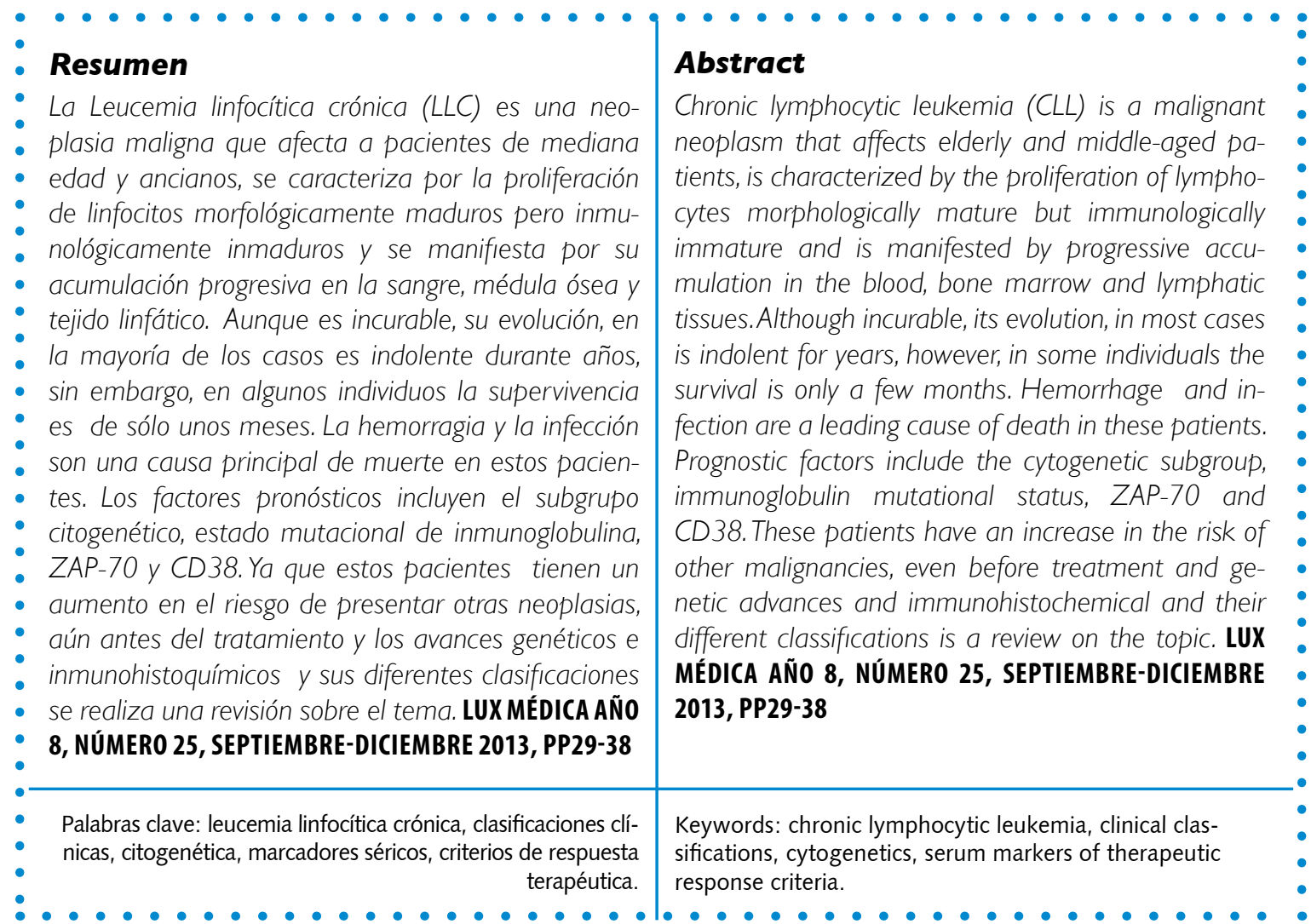

* Médico Interno de Pregrado, Hospital General ISSSTE Aguascalientes, Ags,,

** Médico Internista, Profesor del Internado de Pregrado, Hospital General ISSSTE Aguascalientes, Ags

Fecha de recibido 19 de agosto 2013

Fecha de aceptación: 9 de octubre 2013

Correspondencia: MPSS Jorge Omar Arias Segura, Hospital General Aguascalientes del ISSSTE

Av. Universidad \# 1410. Fraccionamiento San Cayetano, CP 20010, Aguascalientes, Ags., México. Teléfono (01-449) 914-21-55 Correo electrónico dd55_88@ hotmail.com 


\section{Introducción}

\section{Definición y epidemiología}

Clásicamente se ha considerado a la leucemia linfocítica crónica (LLC) como un trastorno proliferativo maligno de linfocitos de aspecto maduro e incompetentes que se acumulan masivamente en sangre, médula ósea y tejidos linfoides. Las primeras descripciones de esta enfermedad fueron hechas en el siglo XIX. ${ }^{1}$ En fechas recientes, la clasificación de las neoplasias hematopoyéticas de la Organización Mundial de la Salud siempre la menciona como una enfermedad neoplásica de las células B, mientras que la entidad antes descrita LLC de linfocitos $T$ ahora es llamada leucemia prolinfocítica de células T. ${ }^{2}$

Representa el $30 \%$ de todas las leucemias en la raza caucásica, siendo la más frecuente en esta población. Por otro lado, la LLC solo abarca el $3-5 \%$ de las leucemias en asiáticos, poniendo esto en evidencia su importante factor racial. ${ }^{3,4}$ En un estudio realizado por el National Cancer Institute's $(\mathrm{NCl})$, se encontró que la incidencia de LLC en cada raza que ha emigrado a los EEUU sigue siendo igual de baja que la reportada en sus respectivos países de origen. ${ }^{5}$ La incidencia en los EEUU es de 3.8 por 100, 000 habitantes al año, ${ }^{6}$ sin embargo, en mayores de 70 años llega a aumentar hasta 12.8 por cada 100,000 personas. ${ }^{7} \mathrm{Su}$ aparición es excepcional antes de los 40 años y su frecuencia aumenta exponencialmente con la edad, de tal manera que la media de presentación es a los 65 años. Su frecuencia es mayor en hombres que en mujeres con una relación de 2:1.4. ${ }^{6}$ En los EEUU, durante el año 2010, se calcularon un total de 14, 990 casos de LLC de los cuales 8,870 fueron hombres y 6,120 fueron mujeres. La mortalidad total fue de 4,390; 2, 650 hombres y 1, 740 mujeres. ${ }^{8}$

En el $90 \%$ de los casos son los linfocitos $B$ las células responsables de producir esta enfermedad, mientras que el escaso $10 \%$ restante tiene su origen en los linfocitos $T$.
De todos los procesos proliferativos de células $T$ esta entidad constituye únicamente el $15 \%$ de los mismos y se ha relacionado con la exposición al human T-lymphotropic virus $1(\mathrm{HTLV}-1)$ el cual es endémico en el suroeste de Japón, África central y en el Caribe. Tiene una supervivencia promedio un poco mayor a dos años. Una característica distintiva de esta entidad es la producción de hematodermias como el síndrome de Sézary (eritrodermia, linfadenopatías y presencia en sangre de células de Sézary). ${ }^{9,10}$

\section{Fisiopatogenia}

Este tipo de cáncer no ha sido posible relacionarlo en su patogénesis con algún tipo de factor ambiental; no así con los factores hereditarios ya que es frecuente encontrar varios casos de esta enfermedad en una sola familia. Debido a esto, los familiares de primera línea de un enfermo con LLC tienen 3 veces más riesgo de presentarla. ${ }^{1}$

Por otro lado, también se han identificado anormalidades genéticas que tienen importancia clínica y pronóstica. La deleción del brazo largo del cromosoma ${ }^{13}$ (13q14-23.1) es la anormalidad más frecuente, se observa hasta en el $50 \%$ de los casos. En el 30\% de ellos se han identificado alteraciones de una región telomérica al gen del retinoblastoma (RB1), hecho implicado en la patogénesis de la enfermedad. Se asocia con un buen pronóstico ya que, en general, hay un largo intervalo entre el diagnóstico y el inicio del tratamiento. ${ }^{6,11}$ La trisomía ${ }^{12}$ se ve en el $10-25 \%$ de los casos. En este cromosoma se regulan oncogenes como CDK2, CDK4, STAT6, APAF-1 y MSM-2 que participan en el ciclo celular y en la apoptosis, se asocia a un pronóstico intermedio y, por lo general, se detecta en casos avanzadas o en los que manifiestan síndrome de Ritchter. La deleción del 17p es menos frecuente, encontrándose en el $10-15 \%$ de los casos. En este cromosoma se encuentra el gen p53 que es un supresor de tumores y que se ve alterado en casi la mitad de los cánceres en seres humanos. Se 
ha asociado con un mal pronóstico ya que tiene manifestaciones clínicas más agresivas así como una alta tasa de resistencia al tratamiento. ${ }^{1,6}$ La deleción del cromosoma 11 (11q22-q23) se reporta en el 12-25\% de los casos. Se asocia clínicamente a pacientes más jóvenes (menores de 55 años) y suelen presentar un estadio clínico más agresivo, con un pronóstico malo. Se relaciona con el gen ATM, el cual tiene la función de reparación del DNA y participa en la patogénesis de formas agresivas de LLC de pobre pronóstico. La deleción del brazo largo del cromosoma 6 (6q21 y 6q24) es una enfermedad más agresiva, se relaciona con cifras elevadas de prolinfocitos en sangre. ${ }^{7}$

Dentro de la patogénesis, también se ha encontrado la deleción o regulación a la baja de dos genes supresores de tumores (miR15 y el miR16 en el cromosoma $\left.13 q 14 .{ }^{3}\right)$ responsables de producir micro RNAs (miRNAs). Los miRNAs son pequeñas moléculas de RNA (menos de 20 nucleótidos) que afectan diversas funciones biológicas. La deleción de estos genes produce niveles disminuidos de miRNAs. Hay una correlación inversa entre miRNA y la expresión de BCL2 (proteína antiapoptótica), y al estar aumentada esta última se produce resistencia a la muerte celular programada. ${ }^{6}$ Reportes recientes han hecho notar que estos miRNAs en plasma son buenos biomarcadores que si se asocian a otros (CD5, CD184, ZAP-70) pueden ofrecer un alto rango de sensibilidad y especificidad en la detección y clasificación de LLC, así como de otros tumores. ${ }^{12,13}$

Se conocen otros factores biológicos que nos pueden ayudar a dar un criterio pronóstico sobre los pacientes que manifiestan esta enfermedad tales como el tiempo de duplicación de los linfocitos, mutaciones de la cadena pesada de inmunoglobulina (IGVH) y la expresión de ZAP70. El tiempo de duplicación de los linfocitos se basa en el hecho de que éstos tienen un índice bajo de mitosis con bajos niveles de p27 (molécula que facilita entrar a la fase $S$ del ciclo celular), hay pacientes con LLC que tienen altos niveles de p27 y por ese motivo hay un rápido incremento de los linfocitos en sangre periférica. Cuando la cifra total es el doble que la del diagnóstico en menos de un año, entonces se habla de una enfermedad en progresión que tiene una supervivencia menor comparada con los pacientes que mantienen rangos de linfocitos más estables.

El receptor intracelular tirosina quinasa ZAP-70 se ha correlacionado bien con las mutaciones del gen de las regiones variables de la cadena pesada de inmunoglobulina (IgVH). En la mitad de los casos las células expresan genes no mutados, mientras que en el resto puede haber grados variables de mutación, en las cuales hay un pronóstico más favorable en comparación de los individuos en los cuales no hay mutaciones. Cuando hay una expresión de ZAP-70 mayor al $20 \%$ asociado a IgVH sin mutaciones indica un pobre pronóstico. Otro factor de mal pronóstico es la expresión del CD38 en más del $30 \%$ de las células leucémicas, lo que se relaciona con una menor supervivencia. 6,14

A partir del año 2001 la OMS incluyó a la leucemia linfocítica crónica (LLC) y al linfoma linfocítico de células pequeñas (LLP) en una sola entidad ${ }^{5}$ en función de que éstas son indistinguibles una de otra en su morfología, fenotipo, genotipo e histopatología. La LLC afecta principalmente a la sangre periférica, mientras que el LLP a los ganglios linfáticos. ${ }^{15}$

La LLC/LLP muestra un inmunofenotipo característico de CD5, CD23 que raramente se identifica en linfocitos $B$ normales. También se puede observar en más del $90 \%$ una disminución de la expresión de las cadenas ligeras de superficie. Con respecto a las cadenas pesadas es habitual encontrar el patrón de expresión IgM/IgD y típicamente funcionan como autoanticuerpos. ${ }^{1,6}$ Morfológicamente las células son pequeños linfocitos con cromatina condensada, núcleos redondos y citoplasma escaso, presentes en sangre periférica, 
médula ósea y ganglios linfáticos. En estos últimos se pueden llegar a observar junto con prolinfocitos, células que típicamente forman agregados llamados centros de proliferación. ${ }^{15-17}$ Debido a su fragilidad durante la preparación del frotis, los linfocitos anormales sufren alteraciones morfológicas dando lugar a las llamadas células difuminadas bajo el microscopio. ${ }^{15}$

\section{MANIFESTACIONES CLÍNICAS}

Es una enfermedad con un curso clínico muy variable, puede haber pacientes asintomáticos durante largos periodos, o bien se pueden presentar formas agresivas y resistentes al tratamiento. En términos generales, al principio es poco agresiva, pudiendo ir desde asintomática o sólo con algunos síntomas inespecíficos tales como fatiga, hiporexia y pérdida de peso. Los signos clínicos que se encuentran con mayor frecuencia son la linfadenopatía y la hepatoesplenomegalia. Los crecimientos ganglionares suelen estar presentes en un $80 \%$ de los pacientes en el momento del diagnóstico, las cuales son de mayor frecuencia a nivel supraclavicular, cervical y axilar. En otras ocasiones, es la linfocitosis la primera manifestación de esta entidad, que por definición debe ser mayor de $5 x$ $10^{9} / \mathrm{L}$, ésta suele ser un hallazgo accidental cuando se practica una biometría hemática de rutina como requisito en trabajos, escuelas. ${ }^{1,18}$ Puede haber casos de infiltración leucémica al sistema nervioso central (SNC), con manifestaciones tales como cefalea, confusión, parálisis de pares craneales, y neuropatías periféricas. Sin embargo esto es excepcional ya que se ha reportado en menos del $1 \%$ de los casos. ${ }^{19}$

En el curso del padecimiento se puede llegar a encontrar cualquier citopenia ya sea por hemólisis autoinmune o por invasión a médula ósea. Esta última se ha cuestionado mucho: Tsopra y cols realizaron un estudio en el que se llegó a la conclusión de que la infiltración a médula ósea no es la única forma de producción de anemia en pacientes con LLC. Asimismo encontró que los niveles séricos de factor de necrosis tumoral alfa (TNF- $\alpha$ ) son mayores en pacientes con anemia relacionada a LLC. ${ }^{20}$ Los glóbulos rojos tienden a ser normocíticos normocrómicos.

También puede haber transformación de LLC a un linfoma difuso de células grandes (síndrome de Richter), el cual es agresivo y se acompaña de un cambio espontáneo en la sintomatología, así como de crecimiento repentino de los ganglios linfáticos. Ocurre en el $5-20 \%$ de los pacientes. ${ }^{21,22}$ También se han descrito inusuales transformaciones a mieloma múltiple y linfoma de Hodking. ${ }^{6}$

\section{Sistemas de clasificación}

1) Criterios de Rai

Este sistema busca dar un criterio pronóstico. Se propuso bajo la premisa de que la LLC es un trastorno progresivo en el que primero se acumulan linfocitos no funcionales inicialmente en sangre y médula ósea, luego invaden ganglios linfáticos, bazo e hígado, y posteriormente resulta en una trombocitopenia, anemia y muerte. ${ }^{23}$

Sistema de clasificación Rai

\begin{tabular}{|ll|}
\hline Estadio & Características \\
\hline O & Linfocitosis. \\
\hline I & Linfocitosis y linfadenopatías \\
\hline II & Linfocitosis con hepatomegalia o esplenomegalia, puede o no haber linfadenopatía. \\
\hline III & Linfocitosis y anemia $(\mathrm{Hb}<11 \mathrm{~g} / \mathrm{dL})$ con o sin linfadenopatía, hepatoesplenomegalia. \\
\hline IV & Linfocitosis y trombocitopenia $\left(<100 \times 10^{9}\right)$ con o sin linfadenopatía, hepatoesplenomegalia. \\
\hline
\end{tabular}


En la actualidad se prefiere utilizar la escala de Rai modificada, en la cual se reducen los cinco estadios originales a tres grupos: Enfermedad de bajo riesgo (estadio Rai 0 ), enfermedad de riesgo intermedio (estadio Rai I o II) y enfermedad de alto riesgo (estadio Rai III o IV). ${ }^{24}$

2) Binet: Es otro sistema con un valor pronóstico bueno, se caracteriza por un menor número de estadios. Sólo requiere de un examen clínico y de una biometría hemática. (25)

\begin{tabular}{|lll|}
\hline Estadio & Características & Supervivencia \\
\hline A & 2 o menos áreas linfoides afectadas* & 10 años \\
\hline B & 3 o más áreas linfoides afectadas* & 7 años \\
\hline C & Anemia $(\mathrm{Hb}<10 \mathrm{~g} / \mathrm{dL})$ y/o trombopenia (plaquetas $\left.<100 \times 10^{9} / \mathrm{L}\right)$ & 2 años \\
\hline
\end{tabular}

* Áreas linfoides afectadas: ganglios cervicales, axilares, inguinales, bazo e hígado.

\section{DIAGNÓSTICO}

A lo largo de la década pasada se obtuvieron grandes avances en el descubrimiento de nuevos marcadores séricos, así como de la utilización de diversos fármacos. El National Cancer Institute $(\mathrm{NCl})$ publicó en 1988 (actualizada en 1996) la guía para el diseño y realización de ensayos clínicos en pacientes con LLC para facilitar las comparaciones entre los diferentes tratamientos, así como para definir aspectos que podrían ser útiles en estudios científicos de esta enfermedad. Esta guía es de importancia, ya que nos es de utilidad en el manejo clínico de los pacientes con LLC, motivo por el cual se mencionarán los puntos principales de dicho documento. ${ }^{24}$

Básicamente, ante un paciente con LLC se deben evaluar el hemograma, el frotis sanguíneo así como el fenotipo inmunológico de las células linfoides circulantes.

- En el hemograma se debe encontrar al menos $5 \times 10^{9}$ linfocitos/L $(5000 / \mu \mathrm{L})$ en sangre periférica de por lo menos tres meses. Estos linfocitos B clonales deben ser confirmados mediante citometría de flujo. En ausencia de adenopatías, visceromegalias, citopenias, o cualquier otro síntoma relacionado con la enfermedad, la presencia de menos de $5 \times 10^{9}$ linfocitos/L se define como "linfocitosis B monoclonal", que puede progresar a un LLC a un ritmo de 1 $2 \%$ por año.

- El frotis se caracteriza por linfocitos maduros, pequeños, con escaso citoplasma y un núcleo denso con cromatina condensada; es posible observar las sombras de Gumprecht. Estas células se encuentran mezcladas con prolinfocitos, que podrán incluir hasta un $55 \%$ de los linfocitos sanguíneos; si este porcentaje es mayor, entonces sugerirá una leucemia prolinfocítica, que en este caso el diagnóstico deberá ser confirmado por histopatología mediante una biopsia de ganglio linfático.

- Inmunofenotipo: Los linfocitos en la LLC expresan los antígenos CD5 y CD23; C D19 y CD20 son coexpresión de los linfocitos $B$ y el CD 5 de las células $\mathrm{T}$; el CD20 y CD79b se encuentran en bajos niveles comparados con los encontrados en las células $B$ normales. En contraste, la mitad de los casos de leucemia prolinfocítica no expresan CD5 y típicamente hay niveles elevados de CD20 e inmunoglobulinas de superficie. ${ }^{24,26}$

Existen otros tipos de pruebas que se pueden utilizar para la evaluación y el diagnóstico de la LLC, sin embargo no son necesarias para establecer el diagnóstico. Con excepción de la hibridación fluorescente 
in situ (FISH), no deben ser utilizados de forma rutinaria y restringir su uso solo en los casos en que se necesite una mejor predicción del progreso de la enfermedad. Por otro lado, la indicación para el inicio del tratamiento no depende de ninguna de estas pruebas, sino de la fase clínica y de la actividad de la enfermedad.

\section{Citogenética}

Mediante el empleo del FISH, se pueden identificar alteraciones citogenéticas en más del $80 \%$ de las LLC. Las deleciones más frecuentes son las del brazo largo de los cromosomas: 13 (13q14.1), 11 (11q) y $6(6 q)$; la del brazo corto del cromosoma 17 (17p) y la trisomía del cromosoma 12. Estas alteraciones tienen significado pronóstico y ayudan a distinguir entre LLC de otros trastornos linfoproliferativos.

\section{Mutación de los genes IgVH y VH3.2I. Uso y expresión del ZAP-70 o CD38.}

Las células leucémicas pueden o no tener mutaciones en los genes de las regiones variables de la cadena pesada de inmunoglobulina (IgVH). Los pacientes sin mutaciones tienen una peor sobrevida comparándose con quienes sí tienen la mutación.

El gen $\mathrm{VH}^{21}$ es un marcador de mal pronóstico de manera independiente a la mutación IgVH. La expresión de ZAP70 o CD38 se correlaciona con la expresión de genes sin mutación IgVH y, en general, traducen un mal pronóstico. ${ }^{24}$

\section{Marcadores séricos}

Se han reconocido numerosas proteínas en sangre que se encuentran elevadas en los pacientes que padecen una forma agresiva de la enfermedad. Los factores más importantes son la CD23 soluble, la timidino kinasa (TK) y la $\beta 2$-microglobulina ( $\beta 2-M G)$. La $\beta 2-M G$ es una proteína extracelular que se asocia con una supervivencia corta de la enfermedad. La TK, en los pacientes con LLC, se relaciona con el grado de proliferación de células leucémicas, además tiene una buena capacidad de detectar a los pacientes con riesgo de un rápido progreso de la enfermedad. El CD23 es una importante molécula de superficie de las células $B$ que indica un mal pronóstico.

A pesar de esto, el valor de estos marcadores como factores pronósticos individuales se encuentra limitado debido a la falta de más ensayos clínicos que lo prueben, es por eso que siempre deben interpretarse bajo la integración de otros métodos de estudio de la LLC. ${ }^{29}$

\section{Examen de médula ósea}

En la LLC más del 30\% de las células nucleadas en el aspirado son linfocitos. Los patrones de infiltración medular pueden ser difuso y no difuso (nodular, intersticial y mixto). Los pacientes con patrón difuso se relacionan con un pobre pronóstico. Dentro de las formas no difusas, los pacientes con patrón intersticial se asocian con un buen pronóstico al igual que las formas nodular y mixta. ${ }^{1}$ Aunque el tipo de infiltración de la médula refleja la carga tumoral y proporciona información pronóstica, hay evidencia actual que sugiere que el valor de una biopsia puede ser reemplazada por nuevos marcadores.

El aspirado y biopsia generalmente no son requeridos para el diagnóstico de LLC, sin embargo ayudan a evaluar las citopenias, éstas se pueden relacionar con varias causas como la infiltración a la médula ósea, ó el uso de fármacos. Es por eso que se recomienda realizar biopsia de médula ósea antes de iniciar el tratamiento. ${ }^{24}$

\section{LINEAMIENTO PARA EL TRATAMIENTO}

El tratamiento de la LLC ha mejorado dramáticamente en los últimos diez años debido al uso combinado de quimioterapia, sin embargo, el régimen óptimo aún no ha sido definido claramente. Las guías 
del "International Worshop on CLL" publicadas en 2008 reemplazaron a las publicadas por el "National Cancer Institute" en 1996. A pesar de esto aún quedan muchas dudas en cuanto a la definición de ciertos criterios, por lo que aún resta mucho trabajo en la elaboración de una referencia más precisa. ${ }^{27}$ A continuación se mencionan los puntos más importantes sobre el tratamiento de la guía publicada en 2008:

\section{Primera línea de tratamiento}

No existe una cura para la LLC. Los pacientes recién diagnosticados asintomáticos con enfermedad en estadio temprano (Rai 0, Binet A) sólo deben ser monitorizados continuamente y no deben ser manejados con quimioterapia ya que no hay evidencias que indiquen que el tratamiento en esta etapa aumente la supervivencia. Únicamente se debe dar medicación en los casos que cursen con progresión rápida de la enfermedad. Un recuento elevado de linfocitos no es indicación de iniciar tratamiento. ${ }^{24,28}$

\section{Indicaciones para iniciar el tratamiento en la LLC1}

\begin{tabular}{|ll|}
\hline$\bullet$ & Anemia, trombocitopenia. \\
\hline$\bullet$ & Síntomas asociados a la enfermedad. \\
\hline$\bullet$ & Esplenomegalia marcada o bazo doloroso. \\
\hline$\bullet$ & Adenopatías sintomáticas. \\
\hline$\bullet$ & Tiempo de duplicación de linfocitos menor a 6 meses. \\
\hline$\bullet$ & Transformación prolinfocítica. \\
\hline$\bullet$ & Transformación Richter. \\
\hline
\end{tabular}

A los pacientes con riesgo intermedio (estadios I y II) y alto (estadios III y IV) de la clasificación de Rai, o los estadios B o $C$ de Binet, por lo general les va bien con el tratamiento. Sin embargo, a pesar de esto, algunos de estos pacientes (Rai riesgo intermedio o Binet fase B) se pueden controlar sin tratamiento hasta que exista enfermedad activa, la cual se define por manifestar alguno de los siguientes puntos:

1) Evidencia de fracaso medular progresivo que se manifiesta por el desarrollo o empeoramiento de anemia $\mathrm{y} / \mathrm{o}$ trombocitopenia.

2) Esplenomegalia ya sea masiva (al menos $6 \mathrm{~cm}$ debajo del reborde costal izquierdo) o progresiva.

3) Linfadenopatía con nódulos masivos (al menos $10 \mathrm{~cm}$ de diámetro mayor) o progresiva.

4) Linfocitosis progresiva con un incremento de más del $50 \%$ en un período de 2 meses o bien, tiempo de duplicación de linfocitos menor de 6 meses.

5) Anemia y/o trombocitopenia poco sensibles a los corticosteroides o a la terapia estándar.

6) Síntomas constitucionales (uno o más de los siguientes):

- Pérdida de peso del $10 \%$ o más en los últimos 6 meses.

- Fatiga significativa (incapacidad para trabajar o realizar actividades cotidianas).

- Fiebre superior a $38.0^{\circ} \mathrm{C}$ durante $2 \mathrm{o}$ más semanas sin evidencia de infección.

- Sudoración nocturna durante más de 1 mes sin evidencia de infección.

Cuando se ha decidido iniciar la terapia se tienen que establecer los objetivos que se desean obtener de la misma, una vez que el problema se resuelve el tratamiento debe detenerse ya que no hay evidencia de 
que la terapia de mantenimiento aumente la supervivencia.

\section{Segunda línea de tratamiento}

En general sigue las mismas indicaciones que en la primera línea. Los pacientes que tienen formas resistentes de la enfermedad, un corto periodo de progresión después del primer tratamiento, $\mathrm{y} / \mathrm{o}$ las deleciones del $17 p$, con frecuencia no responden a la quimioterapia estándar y tienen una supervivencia relativamente corta. ${ }^{1,24}$

El trasplante de células madre hematopoyéticas (TCMH) representa la única opción potencialmente curativa para la LLC, sin embargo los regímenes totalmente ablativos se asocian con morbilidad y mortalidad significativas. El papel exacto del TCMH en el tratamiento estándar de la LLC es todavía indefinido. Según los criterios más recientes, las indicaciones para su empleo incluyen a los pacientes más jóvenes que requieren tratamiento con resistencia primaria al manejo con quimioterapia o en la recaída temprana y con anomalías del gen TP53. Sin embargo, la introducción de nuevas terapias dirigidas con menos morbilidad y respuestas duraderas, puede redefinir las indicaciones de trasplante en la LLC. ${ }^{31}$

\section{Criterios de respuesta al tratamiento:}

1) Remisión completa:

Se requiere de los siguientes criterios con una duración mínima de 2 meses:

- Linfocitos en sangre periférica por debajo de $4 \times 10^{9} / L(4000 / \mu L)$.

- Ausencia de adenopatías significativas.

- Ausencia de hepatomegalia y/o esplenomegalia.

- Ausencia de síntomas constitucionales.

- Recuentos sanguíneos por encima de los siguientes valores: $\sqrt{ }$ Neutrófilos mayores de $1,5 \times 10^{9} / \mathrm{L}(1500 / \mu \mathrm{L})$ sin necesidad de factores de crecimiento exógenos.

$\sqrt{ }$ Plaquetas mayores de $100 \times 10^{9} / \mathrm{L}$ $(100000 / \mu \mathrm{L})$, sin necesidad de factores de crecimiento exógenos.

$\sqrt{ }$ Hemoglobina mayor de $110 \mathrm{~g} / \mathrm{L}$ sin necesidad de transfusión de glóbulos rojos y/o eritropoyetina exógena.

2) Remisión parcial:

Se requiere de los siguientes criterios con una duración mínima de 2 meses:

* Disminución de los linfocitos sanguíneos en un $50 \%$ o más comparado con el valor anterior al tratamiento.

* Disminución de linfadenopatías y hepato-esplenomegalia en un 50\% (por TC o por palpación clínica)

* El recuento sanguíneo debe mostrar uno de los siguientes resultados: $\sqrt{ }$ Neutrófilos mayores de $1,5 \times 10^{9} / \mathrm{L}(1500 / \mu \mathrm{L})$ sin necesidad de factores de crecimiento exógenos.

$\sqrt{ }$ Recuento plaquetario superior a $100 \times 10^{9} / \mathrm{L}(100 \mathrm{000} / \mathrm{uL})$ o bien, el $50 \%$ de mejora sobre la línea de base sin necesidad de factores de crecimiento exógenos.

$\sqrt{ }$ Hemoglobina superior a $10 \mathrm{~g} / \mathrm{L}$ o el $50 \%$ de mejora sobre la línea base sin necesidad de transfusiones de glóbulos rojos y/o eritropoyetina exógena.

3) Enfermedad progresiva:

* Linfadenopatías

* Aumento del tamaño de hígado o bazo en un $50 \%$ o más, o la aparición de novo de hepatomegalia o esplenomegalia.

* Aumento en el número de linfocitos sanguíneos en un $50 \%$ o más con al menos 5,000 linfocitos B por $\mu \mathrm{L}$. 
* Transformación a un tipo histológico más agresivo (por ejemplo el síndrome de Richter).

* Aparición de citopenias (neutropenia, anemia o trombocitopenia). ${ }^{24}$
El diagnóstico diferencial se debe hacer con linfomas como el de células del manto, el folicular, el de la zona esplénica marginal, el de células peludas y con la leucemia prolinfocítica B. ${ }^{30,31}$

\section{Conclusiones}

Actualmente se tiene un mejor conocimiento de la LLC, gracias a estos avances se entienden mejor las diferentes formas clínicas de la LLC, que ayudan a cumplir con los objetivos del tratamiento: mejorar la calidad de vida e incrementar la tasa de sobrevivencia de los pacientes.

\section{Bibliografía}

1. Beutler E, Lichtman $M$, Coller B, Kipps T, Seligsohn U. 2005. Hematología, volumen 2. 6a Ed. Capítulo 98: Leucemia linfática crónica y enfermedades relacionadas.

2. Catovsky D, Ralfkiaer E, Muller-Hermelink HK. 2001. T-cell prolymphocytic leukaemia. In: World Health Organization Classification of Tumours. Pathology and Genetics of Tumours of Haemopoietic and Lymphoid Tissues. IARC Press, Lyon, France, pp. 195196.

3. Degos L, Linch DC, Löwenberg B. 2005. Textbook of malignant haematology. 2a Ed. Italia. 521-539.

4. Montserrat E, Rozman C. Chronic lymphocytic leukemia: Present status. Annals of Oncology. 1995; 6(3): 219-235.

5. Dores GM, Anderson WF, Curtis RE, Landgren O, Ostroumova E, Bluhm EC, Rabkin CS, Devesa SS, Linet MS. Chronic lymphocytic leukemia and small lymphocytic lymphoma: overview of the descriptive epidemiology. British Journal of Haematology. 2007; 139(5): 809-819.

6. His E. The Leukemias of mature lymphocytes. Hematology/Oncology Clinics of North America. 2009; 23(4): 843-871.

7. Crisan D. 2010. Hematopathology: Genomic Mechanisms of Neoplastic Diseases. 1a Ed. Estados Unidos. 256.

8. Jemal A, Siegel R, Xu J, Ward E. Cancer Statistics. CA: A Cancer Journal for Clinicians. 2010; 60(5): 277300.

9. Macon W. Peripheral T-Cell Lymphomas. Hematology/Oncology Clinics of North America. 2009; 23(4): 829-842.

10. Arenas R. 2009. Dermatología. Atlas, diagnostic y tratamiento. $4^{\mathrm{a}}$ edición. México. 754-757

11. Dal Bo M, Rossi FM, Rossi D, Deambrogi C, Berto- ni F, Del Giudice I, Palumbo G, Nanni $M$, Rinaldi $A$, Kwee I, Tissino E, Corradini G, Gozzetti A, Cencini E, Ladetto M, Coletta AM, Luciano F, Bulian P, Pozzato G, Laurenti L, Forconi F, Di Raimondo F, Marasca R, Del Poeta G, Gaidano G, Foà R, Guarini A, Gattei $\mathrm{V}$. $13 q 14$ Deletion size and number of deleted cells both influence prognosis in chronic lymphocytic leukemia. Genes, Chromosomes and Cancer. 2011; 50(8): 633-643.

12. Moussay E, Wang K, Cho JH, Moer K, Pierson S, Paggetti J, Nazarov P, Palissot V, Hood L, Berchem G, Galas DL. MicroRNA as biomarkers and regulators in B-cell chronic lymphocytic leukemia. PNAS. 2010; 108: 6573-6578

13. Fabbri $M$, Bottoni $A$, Shimizu $M$, Spizzo $R$, Nicoloso MS, Rossi S, Barbarotto E, Cimmino A, Adair B, Wojcik SE, Valeri N, Calore F, Sampath D, Fanini F, Vannini I, Musuraca G, Dell'Aquila M, Alder H, Davuluri RV, Rassenti LZ, Negrini M, Nakamura T, Amadori D, Kay NE, Rai KR, Keating MJ, Kipps TJ, Calin GA, Croce CM. Association of a MicroRNA/TP53 Feedback Circuitry With Pathogenesis and Outcome of B-Cell Chronic Lymphocytic Leukemia. JAMA. 2011; 305(1): 59-67.

14. Kern W, Dicker F, Schnittger S, Haferlach C, Haferlach $\mathrm{T}$. Correlation of flow cytometrically determined expression of ZAP-70 using the SBZAP antibody with IgVH mutation status and cytogenetics in 1,229 patients with chronic lymphocytic leukemia. Cytometry Part B: Clinical Cytometry. 2009; 76(6): 385-393.

15. Kumar V, Abbas A, Fausto N. 2005. Patología estructural y functional.7a Ed. España. 677- 678.

16. Nabhan C. 2007. Chronic Lymphocytic Leukemia Research Focus. 1a Ed. Estados Unidos. 1-10.

17. Linet MS, Schubauer-Berigan $M K$, Weisenburger DD, Richardson DB, Landgren O, Blair A, Silver S, Field RW, Caldwell G, Hatch M, Dores GM. Chronic lymphocytic leukaemia: an overview of aetiology 
in light of recent developments in classification and pathogenesis. British Journal of Haematology. 2007; 139(5): 672-686.

18. Redaelli A, Laskin B, Stephens J, Botteman M, Pashos $C$. The clinical and epidemiological burden of chronic lymphocytic leukaemia. European Journal of Cancer Care. 2004; 13(3): 279-287.

19. Denier C, Tertian G, Ribrag V, Lozeron P, BilhouNabera C, Lazure T, Abbed K, Lacroix C, Adams D. Multifocal deficits due to leukemic meningoradiculitis in chronic lymphocytic leukemia. Journal of the Neurological Sciences. 2009; 277(1-2): 130-132.

20. Tsopra OA, Ziros PG, Lagadinou ED, et al. Diseaserelated anemia in chronic lymphocytic leukemia is not due to intrinsic defects of erythroid precursors: A possible pathogenetic role for tumor necrosis factoralpha. Acta Haematológica. 2009; 121(4): 187-195.

21. Ossi D, Cerri M, Capello D, Deambrogi C, Rossi FM, Zucchetto A, De Paoli L, Cresta S, Rasi S, Spina V, Franceschetti $S$, Lunghi $M$, Vendramin $C$, Bomben $R$, Ramponi A, Monga G, Conconi A, Magnani C, Gattei V, Gaidano G. Biological and clinical risk factors of chronic lymphocytic leukaemia transformation to Richter syndrome. British Journal of Haematology. 2008; 142(2): 202-215.

22. Deambrogi C, Cresta S, Cerri M, Rasi S, De Paoli L, Ramponi A, Gaidano G, Rossi D. 14q32 Translocations and risk of Richter transformation in chronic lymphocytic leukaemia. British Journal of Haematology. 2009; 144(1): 131-133.

23. Rai KR, Sawitsky A, Cronkite EP, et al. Clinical staging of chronic lymphocytic leukemia. Blood. 1975; 46(2): 219-34.

24. Hallek M, Cheson BD, Catovsky D, Caligaris-Cappio $F$, Dighiero $G$, Döhner $H$, Hillmen $P$, Keating $M J$, Montserrat E, Rai KR, Kipps TJ. Guidelines for the diagnosis and treatment of chronic lymphocytic leu- kemia: A report from the International Workshop on Chronic Lymphocytic Leukemia updating the National Cancer Institute-Working Group 1996 guidelines. Blood. 2008; 111(12): 5446-5456.

25. Binet JL, Auquier A, Dighiero G, Chastang C, Piguet $\mathrm{H}$, Goasguen J, Vaugier $\mathrm{G}$, Potron $\mathrm{G}$, Colona $\mathrm{P}$, Oberling F, Thomas M, Tchernia $G$, Jacquillat $C$, Boivin $P$, Lesty $C$, Duault $M T$, Monconduit $M$, Belabbes $S$, Gremy F. A new prognostic classification of chronic lymphocytic leukemia derived from a multivariate survival analysis. Cancer. 1981; 48(1): 198-206.

26. Eichhorst $B$, Hallek $M$, Dreyling $M$. Chronic lymphocytic leukaemia: ESMO Clinical Practice Guidelines for diagnosis, treatment and follow-up. Annals of Oncology. 2010; 21(5): 162-164

27. Foon KA. Clearly defined response criteria evaluating treatment of CLL patients in clinical research trials. Blood. 2010; 116(10): 1817-1818.

28. Dighiero G, Maloum K, Desablens B, Cazin B, Navarro $M$, Leblay $R$, Leporrier $M$, Jaubert J, Lepeu $G$ Dreyfus $B$, Binet $J$, Travade P. Chlorambucil in indolent chronic lymphocytic leukemia. French Cooperative Group onChronic Lymphocytic Leukemia. The New England Journal of Medicine. 1998; 338(21): 1506-1514

29. Montillo M, Hamblin T, Hallek M, Montserrat E, Morra E. Chronic lymphocytic leukemia: novel prognostic factors and their relevance for risk-adapted therapeutic strategies. Hematologica/The Hematology Journal. 2005; 90(3): 391-399.

30. Vyas N, Hassan A. Recent advances in chronic lymphocytic leukemia. Indian Journal of Cancer. 2012 49(1): 137-143.

31. Smolewski P, Witkowska $M$, Korycka-Wolowiec A. New Insights into Biology, Prognostic Factors, and Current Therapeutic Strategies in Chronic Lymphocytic Leukemia. ISRN Oncology. 2013; 2013(1): 1-7 